\title{
Asthma admissions, smoking bans and administrative databases: author's response
}

The issue of the potential misclassification within Hospital Episode Statistics (HES) data of asthma for another condition such as COPD, or vice versa, raised by Kent et $a l^{1}$ is an important one and any impact that this may have should be considered. Misclassification with resultant bias is a potential risk of many studies. However, the extent, or direction, to which bias may be introduced to a study that uses the recorded primary diagnosis from nationally collected data to ascertain trends in admissions is difficult to estimate.

Kent et al' claim that asthma is misclassified in $9-14 \%$ of cases is based on a single study $^{2}$ using data, which is now at least 20 years old and came from only two hospitals in one region of England. As such it is highly unlikely to be representative of current national coding practice. Routinely collected data sets such as HES are continuously audited and the accuracy of clinical coding is assessed. We note that recent studies, although not specific to asthma or indeed other respiratory conditions, report that the introduction of measures such as Payment by Results has led to an improvement in the quality of coding in HES, ${ }^{3}{ }^{4}$ and that 'current levels of reported accuracy suggest that routinely collected data are sufficiently robust to support their use for research and managerial decisionmaking. ${ }^{4}$ A 2008/09 audit of all acute National Health Service trusts in England reported a mean coding error for any primary diagnosis of $13.1 \%,{ }^{5}$ considerably smaller than the $28 \%$ and $45 \%$ reported by the two hospitals in the Dixon et $a l^{2}$ study for any primary diagnosis suggesting there have been marked improvements in coding accuracy over time.

The use of the primary diagnosis from the HES data set is an established method of analysis ${ }^{6}$ to establish trends 
in emergency or elective admissions and is routinely used for this purpose. ${ }^{8}$ While we acknowledge the potential issue of misclassification using the primary diagnosis of a patient's stay in hospital and the possibility that our study may, therefore, be subject to some bias, we note that it is only the coding error rates around the time of the legislation that will impact on our findings. These error rates though are likely to be considerably smaller than those quoted by Kent et al based on the Dixon et $a l^{2}$ study. Moreover, the direction and magnitude will further depend on the differential effect that secondhand smoke has on asthma compared with the other conditions misclassified as asthma about which very little is known.

\section{Michelle Sims, ${ }^{1}$ Roy Maxwell, ${ }^{2}$ Anna Gilmore ${ }^{1}$}

${ }^{1}$ School for Health, and the UK Centre for Tobacco Control Studies, University of Bath, Bath, UK

${ }^{2}$ Public Health England, Bristol, UK

Correspondence to Dr Michelle Sims, Tobacco Control Research Group, Department for Health, University of Bath, Claverton Down, Bath BA2 7AY, UK; m.sims@bath.ac.uk

Contributors MS, RM and AG drafted the letter.

Funding The original work was undertaken by the University of Bath which received funding from the Department of Health's Policy Research Programme.

Competing interests $M S$ and $A G$ are members of the UK Centre for Tobacco Control Studies which receives core funding from the British Heart Foundation, Cancer Research UK, Economic and Social Research Council, Medical Research Council, and the National Institute of Health Research under the auspices of the UK Clinical Research Collaboration.

Disclaimer The views expressed in the publication are those of the authors and not necessarily those of the Department of Health.The funders had no role in the study design, data collection and analysis, interpretation of data, decision to publish, or preparation of the manuscript.

Provenance and peer review Not commissioned; internally peer reviewed.

To cite Sims M, Maxwell $R$, Gilmore A. Thorax 2013;68:1166-1167.

Received 19 August 2013

Accepted 23 August 2013

Published Online First 16 September 2013

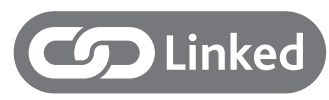

http://dx.doi.org/10.1136/thoraxjnl-2013-204183

Thorax 2013;68:1166-1167.

doi:10.1136/thoraxjnl-2013-204371

\section{REFERENCES}

1 Kent B, Lane S, Moloney E. Asthma admissions, smoking bans, and administrative databases. Thorax 2013:68:1166.
2 Dixon J, Sanderson C, Elliott P, et al. Assessment of the reproducibility of clinical coding in routinely collected hospital activity data: a study in two hospitals. J Public Health Med 1998;20:63-9.

3 Audit Commission. Right data, right payment. Annual report on the payment by results data assurance programme 2011/2012, 2012. http://archive. audit-commission.gov.uk/auditcommission/health/ paymentbyresults/reportsandstudies/Pages/ rightdatapbr2012.aspx.html (accessed 14 Jul 2013).

4 Burns EM, Rigby E, Mamidanna R, et al. Systematic review of discharge coding accuracy. J Public Health 2012;34:138-48.

5 Audit Commission. Payment by results data assurance framework 2008/09. Supplementary analysis. 2009. http://archive.audit-commission.gov.uk/ auditcommission/health/paymentbyresults/ reportsandstudies/pbrdataassuranceframework200809/ pages/default_copy.aspx.html (accessed 14 Jul 2013).

6 Millett C, Lee JT, Laverty AA, et al. Hospital admissions for childhood asthma after smoke-free legislation in England. Pediatrics 2013;131:e495-e501.

7 NHS Atlas of Variation in Healthcare for People with Respiratory Disease. Reducing unwarranted variation to increase value and improve quality. 2012. http:// www.rightcare.nhs.uk/index.php/atlas/ respiratorydisease/ (accessed 14 Jul 2013).

8 Asthma UK. Health and Social Care Bill. Memorandum submitted by Asthma UK (HS 34). 2011. http://www. publications.parliament.uk/pa/cm201011/cmpublicl health/memo/m34.htm (accessed 14 Jul 2013). 